Autores (p.o. de firma): V. Andreu, P. Bazquez-Roig, C. Blasco y Y. Picó

Título: "Determination of tetracycline residues in soils by pressurized liquid extraction and liquid chromatography tandem mass spectrometry"

Revista: Analytical and Bioanalytical Chemistry

Volumen: 394(5) Páginas, inicial: 1329 final: $1339 \quad$ Fecha: 2009 


\section{Determination of tetracycline residues in soil by pressurized liquid extraction and liquid chromatography tandem mass spectrometry}

Vicente Andreu • Pablo V Roig · Cristina Blasco · Yolanda Picó

P.V. Roig, C. Blasco · Y. Picó $(\bowtie)$

Laboratori de Bromatologia, Facultat de Farmàcia, Universitat de València, Av, Vicent Andrés s/n, 46100

Burjassot, València, Spain.

E-mail: yolanda.pico@uv.es

Tel:+34 963543092

Fax:+34 963544954

V. Andreu

Centro de Investigaciones sobre Desertificacion (CIDE), Camí de la Marjal s/n, 46470 Albal, Valencia, Spain E-mail: Vicente.andreu-perez@uv.es

Tel: +34 961220540

Fax: +34 961270967 
Abstract An optimized extraction and clean-up method for the analysis of chlortetracycline (CTC), doxycycline (DC), oxytetracycline (OTC), and tetracycline (TC) in soil is presented. Soil extraction in a pressurized liquid extraction (PLE) system, extract clean-up by solid-phase extraction (SPE) and tetracycline determination by liquid chromatography tandem mass spectrometry (LC-MS/MS) provided appropriate efficiency and reproducibility. Different dispersing agents and solvents for soil extraction and several SPE cartridges for clean-up were compared. The best extraction results were obtained using EDTA treated sand, as dispersing agent, and water at $70^{\circ} \mathrm{C}$. The most effective clean-up was obtained using $\operatorname{StrataX}(\mathrm{TM})$ sorbent in combination with a strong anion exchange cartridge. Recoveries ranged from 70 to $99 \%$ and precision, as indicated by the relative standard deviations (RSDs), was within the range of $8-15 \%$. The limits of quantification (LOQs) by using LC-MS/MS, based on S/N: 10 , ranged from $1 \mu \mathrm{g} \mathrm{kg}^{-1}$ for TC to $5 \mu \mathrm{g} \mathrm{kg}^{-1}$ for CTC. These results pointed out that this technique is appropriate to determine tetracyclines in soils. Analysis of 100 samples taken in the Valencian Community revealed that in soil up to $5 \mu \mathrm{g} \mathrm{kg}{ }^{-1} \mathrm{CTC}, 15 \mu \mathrm{g} \mathrm{kg}^{-1}$ OTC, $18 \mu \mathrm{g} \mathrm{kg}^{-1} \mathrm{TC}$ and $12 \mu \mathrm{g} \mathrm{kg}^{-1}$ DC could be detected. Detection of the analytes in several samples, which typify the Spanish agricultural soils, should be outlined as most important result of this study.

Keywords Antibacterials - Tetracyclines - LC-MS/MS - Pressurized Liquid Extraction · Environmental analysis· Soil 


\section{Introduction}

Pharmaceutical residues in the environment are of increasing concern worldwide because the large number of drugs used in human and veterinary medicine [1-4]. After excretion, these drugs and their metabolites reach the environment by passing sewage treatment plants or by soil amended with sewage sludges or manures [5-7]. New investigations show that more than 45 different drugs can be found in surface waters from the low to the very low $\mu \mathrm{g} 1^{-1}$ concentration range [8-10].

One among the first groups of antibiotics to come into use in human being was the tetracyclines (TCs), these drugs have stood the test of time and are continuing to be useful in treating a broad range of infections [11]. TCs have been found widely disseminated in water and sewage. However, little is know about their occurrence and impact in soils. Some data extracted from the literature show that residues of the commonly used veterinary drugs, tetracycline (TC) and chlortetracycline (CTC) can be detected in soil surface $(0-40 \mathrm{~cm})$ fertilized with animal slurry [12-15]. Nevertheless, no leaching of TCs into deeper soil segments or groundwater has beem observed $[14,15]$. The halflife of oxytetracycline (OTC) in manure was 30 days and the compound was still detectable in this matrix after 5 months. In the manured soils, OTC was detected at concentrations at least 10 times lower than the European Agency for the Evaluation of Medicinal Products threshold (100 $\mu \mathrm{g} \mathrm{kg}^{-1}$ ) [16].

Detection of TCs in soil, sediments, and water, and the growing concern of their potentially adverse effect on natural ecosystems have resulted in a need to understand their behavior in the soil system. TCs have multiple ionizable functional groups such that at environmentally relevant $\mathrm{pH}$ values, they may exist as a cation, zwitterion, or a net negatively charged ions, which complicates predicting their sorption, availability, and transport. The sorption of OTC, TC, and CTC by several soils varying in $\mathrm{pH}$, clay content and type, cation exchange capacity (CEC), anion exchange capacity (AEC), and soil organic carbon was investigated. Strongest sorption was observed for clays, 
followed by HS, and then clay-HC $[17,18]$. Greater sorption by the Ca systems than the K ones and decreased sorption with increasing $\mathrm{pH}$ suggests that cation binding and cation exchange contribute to sorption [19-21].

Summarizing, TCs enter in the environment in significant concentrations via repeated amendments with manure or sludges, build up persistent residues, and accumulate in soil. Therefore, TCs may have a potential risk and investigations on their environmental effects are necessary [22].

In a recent review, O’Connor and Aga [7] discuss strategies for sample preparation, extraction, clean-up using solid-phase extraction (SPE) and molecularly-imprinted polymers (MIPs), and analysis of TCs and their transformation products in soils. This review also points out that available information about the environmentally-relevant concentrations of TCs is limited, mostly due to analytical difficulties encountered when trying to analyze trace levels of these compounds in the presence of complex matrices. Liquid chromatography-tandem mass spectrometry (LC-MS/MS) has become widely used in detecting antibiotics, including TCs. In applying LC-MS/MS for environmental investigations, the analyst is faced with two major challenges: poor detectability of TCs and highly variable matrix interferences, which compromise quantification. Efforts have been directed to attain high-throughput methods able to extract a large number of samples in a short time $[15,16,23]$. Pressurized liquid extraction (PLE) is a rather new technique that uses solvent at a relatively high pressure and temperature without their critical point being reached. This improves efficiency compared to extractions at room temperature and atmospheric pressure [7]. Recently, O'Connor et al. [24] optimized the extraction of TCs by using rapid and simple PLE procedures with a mixture of acetate buffer ( $\mathrm{pH} 8)$, and methanol as the extracting solvent in soil. In the same way, Jacobsen et al. [25] reported the use of PLE with mixtures of methanol and citric acid (pH 4.7) to extract TC, macrolide and sulfonamide antibiotics from agricultural soils. Other studies have reported the use PLE to extract both, veterinary drugs from food matrices [26-28] or different 
contaminants from soils $[29,30]$. All these studies remarked the technology's benefits in providing rapid and reliable analysis.

The present study focuses on developing a method for the simultaneous determination of four TCs — TC, OTC, CTC and doxycycline (DC) — in soil, using PLE and SPE extraction followed by LC-MS/MS with a triple quadrupole (QqQ) analyzer. Different dispersing agents and solvents for soil extraction and several SPE cartridges for clean-up were compared. The method was applied to soil samples of different locations and after different sludge treatments. To the best of our knowledge, this is the first finding of TC residues in typical Spanish agricultural soils.

\section{Experimental}

Chemicals and standards

TC, OTC, CTC, DC and demeclocycline (DMC) were purchased from Sigma (St. Louis, MO). DMC was used as internal standard (IS) because it is an obsolete antibiotic. The three epimers, 4epitetracycline (e-TC), 4-epioxytetracycline (e-OTC), and 4-epichlortetracycline (e-CTC), were from Acros (Fisher Scientific, Schwerte, Germany). HPLC-grade methanol was purchased from Merck (Darmstadt, Germany). Formic acid, citric acid monohydrate, sodium acetate anhydrous, sodium hydroxide pellets and ethylene diaminetetraacetic disodium salt (EDTA- $\left.\mathrm{Na}_{2}\right)$ were of analytical grade (Aldrich, Madrid, Spain). Deionised water $(<8 \mathrm{M} \Omega \mathrm{cm}$ resistivity) was obtained from the Milli-Q SP Reagent Water system (Millipore, Bedford, MA, USA). All the solvents and solutions were filtered through a $0.45 \mu \mathrm{m}$ cellulose filter from Scharlau (Barcelona, Spain) before use. Acidic, neutral and basic alumina $\left(\mathrm{Al}_{2} \mathrm{O}_{3}\right)$ were obtained from Merck, silica gel form scharlau, 
Florisil ${ }^{\circledR}$ from Aldrich, sea sand from Panreac and anhydrous sodium sulfate (analytical grade) from Scharlau. To block metal impurities, $60 \mathrm{~g}$ of solid sorbent was placed in a Buchner funnel and $120 \mathrm{~mL}$ of $0.1 \mathrm{M}$ EDTA-Na $\mathrm{N}_{2}$ was passed through the sorbent using vacuum. SAX cartridges (strong anion exhange, $500 \mathrm{mg}$ sorbent, $6 \mathrm{ml}$ cartridge) were purchased from Isolute, IST. Oasis ${ }^{\circledR}$ HLB extraction cartridges [poly(divinylbenzene-co-N-pyrrolidone), $6 \mathrm{cc}, 200 \mathrm{mg}$ ] were from Waters (Milford, MA, USA) and Strata- $\mathrm{X}^{\circledR}$ SPE cartridges [surface modified styrene divinylbenzene, $6 \mathrm{cc}$, $200 \mathrm{mg}$ ) from Phenomenex (Torrance, CA, USA).

Sample collection

Samples of soil were collected from the Ap horizon $(0-20 \mathrm{~cm})$ from 50 fields located in the South surrounding area of the Valencia city (Spain) in early February 2007. The soils are of loamy texture highly carbonated, representing typical Spanish agricultural soils. Some soil characteristics are listed in Table 1. The last fertilization with sewage sludges was in September 2006 according to the data provided by the Conselleria de Medio Ambient. There were no more data on the amount of sludge added to the field or the contamination levels of the sludges by these pharmaceuticals. Two control samples from fields without slurry fertilization since, at least, 5 years were also taken from this region. Samples were taken in plastic bags and immediately transported under cooling to the laboratory. To achieve homogeneous samples, the soils were air dried and sieved through a $2 \mathrm{~mm}$ sieve before further handling.

Sample preparation 


\section{Pressurized liquid extraction (PLE)}

The extraction of antibacterial agents from soil was performed by PLE, using an ASE 200 system from Dionex (Sunnyvale, CA, USA). The system was operated with pressure resistant steel extraction cells with a volume of $22 \mathrm{ml}$ and lined with glass-fibre filters from Dionex.

Approximately $5 \mathrm{~g}$ of soil were added of $10 \mu \mathrm{l}$ DC (IS) solution of $10 \mu \mathrm{g} \mathrm{ml}^{-1}$ and blended with $5 \mathrm{~g}$ of EDTA-Na $\mathrm{N}_{2}$ washed sea sand for 5 minutes in a mortar using a pestle. This mixture was introduced into a stainless steel extraction cell, which was positioned in the PLE system connected to a four-bottle solvent controller. Nitrogen, at a pressure of $10 \mathrm{bar}$, was supplied to assist the pneumatic system and to purge the extraction cells. The extraction cells were preheated for $2 \mathrm{~min}$, the analytes were extracted with water at $70^{\circ} \mathrm{C}$ and 1500 psi for $10 \mathrm{~min}$ of static time, in one cycle, at $100 \%$ of flush; then, the extraction cells were purged for $60 \mathrm{~s}$ with nitrogen to eliminate any trace of the extraction solvent. The total final volume of extract was approximately $40 \mathrm{ml}$.

\section{Solid-phase extraction}

Clean up and pre-concentration was performed using a combination of SAX and Strata-X cartridges. Cartridges were placed in tandem to simultaneously remove negatively charged humic material (SAX) and retain the antibacterial agents (Strata-X). The SAX cartridge was placed on top of the Strata-X cartridge and both columns were conditioned first with $2 \mathrm{ml}$ methanol and then $2 \mathrm{ml}$ water. PLE extracts $(40 \mathrm{ml})$ were passed through both SPE-columns at approximately $5 \mathrm{ml} \mathrm{min}{ }^{-1}$ and after extraction the columns were washed with $2 \mathrm{ml}$ water and dried under vacuum for $15 \mathrm{~min}$. Then, the SAX cartridge was removed and the antibacterial agents were eluted from the Strata-X sorbent with $2 \mathrm{ml}$ methanol. The eluate was evaporated to dryness using a multi-sample Turbovap LV Evaporator (Zymark, Hoptkinton, MA, USA), and the residue was redissolved in $1 \mathrm{ml}$ methanol-water (10:90). 


\section{Liquid chromatography-mass spectrometry}

A Quattro LC triple quadrupole mass spectrometer from Micromass (Manchester, U.K.), equipped with an LC Alliance 2690 system (Waters) consisted of an autosampler and a quaternary pump, a pneumatically assisted electrospray probe, a Z-spray interface and a Mass Lynx NT software Ver. 4.1 were used. Analysis was performed in positive ion mode. The ESI source values were: capillary voltage, $3.00 \mathrm{kV}$; extractor, $2 \mathrm{~V}$; RF lens, $0.5 \mathrm{~V}$; source temperature, $120{ }^{\circ} \mathrm{C}$; desolvation temperature, $300{ }^{\circ} \mathrm{C}$, and desolvation and cone gas (nitrogen $99,99 \%$ purity) flows, 600 $1 \mathrm{~h}^{-1}$ and $601 \mathrm{~h}^{-1}$, respectively. The analyzer settings were resolution, 12.0 (unit resolution) for the first and third quadrupoles; ion energy, 2.0; entrance and exit energies, -1 and 1; multiplier, 650; collision gas (argon, $99.995 \%$ ) pressure $2.79 \times 10^{-3}$ mbar; interchannel delay, $0.02 \mathrm{~s}$; total scan time, $1.0 \mathrm{~s}$. The mass spectrometer was operated in scan and product ion scan modes to optimize the conditions and select the transitions, and in selected reaction monitoring (SRM) mode to confirm the identity of analytes in the samples by selecting two transitions for each one and to quantify. Table 2 shows the particular conditions and transitions for each analyte. The analytical column was a Xterra $\mathrm{C}_{18}(100 \times 2.1 \mathrm{~mm}$ I.D., $3.5 \mu \mathrm{m})$ from Waters. The mobile phase consisted of methanol and water, both with $10 \mathrm{mM}$ formic acid at $0.2 \mathrm{ml} \mathrm{min}^{-1}$ in gradient that begins with $10 \%$ methanol, increasing linearly in $15 \mathrm{~min}$ to $90 \%$ of methanol, maintaining this proportion for $5 \mathrm{~min}$ and return to the initial conditions in $10 \mathrm{~min}$. The injected volume was $20 \mu 1$.

Method validation 
Recoveries for the entire procedure were determined using the two control samples taken from fields without fertilization. Soil samples were fortified with CTC, OTC, TC and DC on three concentration levels (approximately 10, 50 and $100 \mu \mathrm{g} \mathrm{kg}^{-1}$ soil) and the IS at fixed concentration of $100 \mu \mathrm{g} \mathrm{kg}^{-1}$. Six different extractions were performed at each level. The fortified samples were extracted and analysed using the entire procedure. Recoveries were calculated as the percentage of extracted antibacterial agent compared to the spiked level.

Experiments were performed to determine recoveries of the antibacterial agents CTC, OTC, TC and DC for the tandem SPE (SAX+Strata-X) clean-up step only. Sample matrix was obtained by extracting control samples, using the described PLE method. These PLE extracts were fortified with antibacterial agents at two concentration levels (1.25 and $\left.12.5 \mu \mathrm{g}^{-1}\right)$, corresponding to extracts obtained from extraction of soil samples with antibacterial agent contents of approximately 10 and $100 \mu \mathrm{g} \mathrm{kg}^{-1}$ soil, respectively. The fortified samples were passed through the SAX-Strata.X SPE cartridges as described above.

Day-to-day variations for the extraction procedure were determined, by repeating the recovery experiment for concentration levels of 10,50 and $100 \mu \mathrm{g} \mathrm{kg}^{-1}$ soil after 3 days.

The linearity of the analytical methods was proved building the calibration curves for each compound, using soil extracts without TCs spiked at six concentration levels $(10,25,50,75,100$ and $500 \mu \mathrm{g} \mathrm{kg}^{-1}$ soil) with each TC. Each level was prepared in triplicate. The linear regression analysis was carried out by plotting the peak area ratio of analyte and IS versus the analyte concentration.

Furthermore, the linearity range for the entire soil extraction procedure was determined by fortified soil samples at the same six concentration levels that previously. Triplicate samples were made for concentration levels. 
The LOD was estimated at a signal-to-noise ratio of 3, while the LOQ value was estimated by using a signal-to-noise ratio of 10 . LODs and LOQs were obtained by the transition with higher signal/noise in SRM mode. For the LOQ, the confirmatory transition should be, at least, visible in the chromatogram. Once evaluated, three samples were spiked at the estimated levels and extracted according to the proposed procedure to ensure their feasibility.

\section{Results and discussion}

Soil extraction method optimisation

Extraction solvent for extracting the TCs from soil was selected in accordance with their physicochemical properties. Many different soil-adsorption mechanisms, such as hydrophobic interactions, hydrogen bonding, complexation and cation exchange, may affect the extraction of the compounds from soil $[18,22,31]$. TCs form strong complexes with di- and trivalent cations in the clay mineral inter-layers or with hydroxy-groups at the surface of the soil particles [19-21]. As a starting point, a sample pre-treatment by homogenization of soil with EDTA-Na $\mathrm{Na}_{2}$ washed sand as dispersing agent followed by PLE using water as extractant, was applied as previously employed for food analysis $[27,32]$.

The influence of the sorbent used for the homogenization and dispersion of soil was investigated by performing the homogenization with alumina (neutral and basic), Florisil ${ }^{\circledR}$, silica and sea sand, all of them washed with EDTA-Na $\mathrm{N}_{2}$. The washing of the material was carried out because previous studies performed in food demonstrated that EDTA-Na $a_{2}$ washed materials always provide better recoveries [27]. EDTA- $\mathrm{Na}_{2}$ deactivates metal impurities present in the surface and, probably, 
chelates also metals present in soil facilitating the TCs decomplexation. Silica and sea sand provided the better recoveries for all TCs (up to $40 \%$ ). Alumina, neutral or acid, does not recover these compounds and Florisil ${ }^{\circledR}$ provides good recoveries for OTC, CTC and their epimers but fails in recovering TC, e-TC and DC.

In order to obtain the highest possible concentrations of the TCs in soil extracts, the performance of the PLE-system was investigated for soil samples weighing up to $25 \mathrm{~g}$. However, samples greater than $5 \mathrm{~g}$ were difficult to analyze owed to the larger number of matrix producing peaks. To increase contact-surface between soil particles and water and prevent metal complexation, as well as clogging of the extraction cell, $5 \mathrm{~g}$ EDTA-Na $\mathrm{N}_{2}$ washed sea sand was mixed into $5 \mathrm{~g}$ soil sample before extraction. In development and optimisation of the PLE method several settings need to be considered such as pressure, temperature and number of solvent cycles. The final operating parameters for the validation of the method are listed in the Experimental section (see PLE extraction).

Chemical transformation processes of TCs, such as isomerization and epimerization, have been reported giving rise to structurally related compounds [7]. The possible inter-conversion between TCs and their 4-epimeric (4-eTCs) forms during the extraction procedure was checked by spiking different soil samples only with the TCs or with the 4-eTCs. No inter-conversion between the TCs and its 4-epimers was observed, even through the extraction was performed at $70{ }^{\circ} \mathrm{C}$.

The method was compared with two methods based on PLE recently reported by Jacobsen et al. [25] and O'Connor et al. [24] (Fig 1). The former procedure is based on mix $10 \mathrm{~g}$ soil sample with $10 \mathrm{~g}$ Ottawa sand before added to the extraction cell of $33 \mathrm{ml}$. The extraction buffer consisted of a 50:50 (v/v) mixture of methanol and $0.2 \mathrm{M}$ citric acid in water with $\mathrm{pH}$ adjusted to 4.7 with $\mathrm{NaOH}$. Extractions were performed at room temperature to avoid that the TCs were converted to their epior anhydro form. Recoveries, achieved using this method, were above $40 \%$, which were comparable to recoveries for the proposed combination. The latter protocol involves extraction of $5 \mathrm{~g}$ of soil 
with 50:50 (v/v) methanol-acetate buffer $(\mathrm{pH} 8)$. The percent recoveries of the optimized PLE method varied between the soils and ranged from 22-99\%, depending on soil type, and more specifically on clay content. Comparing the three extraction methods, i.e., the results shown in Fig. 1, all of them provided acceptable recovery levels of the studied TCs with overall recoveries of 77 $\%$ for the method proposed by Jacobsen et al. [25], $74 \%$ for that proposed by O'Connor et al. [24] and $78 \%$ for the method developed in this study. The main advantages of the proposed procedure for the TC extraction from soils are that only uses water as extractant and the stability of the compounds through the extraction procedure.

Clean-up and pre-concentration using SPE

Solid phase extraction (SPE) is traditionally used as a clean-up technique for extracts of environmental samples. The two reported procedures for tetracyclines used combination of SAX and HLB (polymeric) or SAX and StrataX SPE sorbents, respectively. These sorbents act as both clean-up and pre-concentration. The SAX column reduces matrix interferences by adsorbing anionic humic particles from the soil extracts, avoiding contamination, blocking and overloading of the HLB or StrataX sorbent. At the extraction $\mathrm{pH}$, the TCs are overall neutral or cationic and are therefore not retained on the SAX cartridge, while the polymer based HLB or StrataX cartridge simultaneously retains neutral polar and non-polar compounds, including the studied TCs. A study of the results obtained using HLB and Strata X cartridges, individually and the combination SAX and HLB and SAX and StrataX was carried out. HLB and StrataX cartridges, showed, as expected, an appropriate analyte isolation and strong adsorption of matrix compounds, since TCs were quantitatively eluted with $2 \mathrm{ml}$ of methanol. The extract obtained using either HLB or StrataX cartridges showed that extract still has an intense yellow-brown colour indicative of co-extraction of 
soil components. Additional purification of the sample by SAX resulted in a considerable decrease in the humic acid content providing almost transparent extracts. The interpretation of the chromatograms with low concentrations of the analytes improved at the same time as the lifetime of the LC column was prolonged.

Recoveries and corresponding 95\% confidence intervals for the different SPE platforms, at two concentration levels (approximately 1.2 and $12.5 \mu \mathrm{g} 1^{-} 1$ in the final extract) are shown in Fig. 2. The recoveries obtained using the SAX-StrataX systems were slightly better than those obtained with the SAX-HLB because of this, the former was selected for the definitive method.

Validation of the soil extraction procedure

Figs. 3 and 4 display a typical total ion current (TIC) and mass chromatograms obtained for a soil sample spiked with the four TCs plus the IS and with the three 4-epimers and the IS, respectively. The TIC chromatogram is a summation of the ion signal generated by all the precursor $\rightarrow$ product ions transition showed in Table 1. Chemical transformation processes of tetracyclines, such as isomerization and epimerization, have been reported giving rise to structurally related compounds. For instance, CTC is converted to isochlortetracycline (iCTC) under alkaline conditions, while the epimerization has been found to be catalyzed in acidic solutions in a pH range from 2 to 6 [7]. The mass chromatograms (Fig. 1B-E and Fig. 2B-D) show that there is no inter-conversion between TCs and their 4-epimeric forms during the extraction procedure. In the present study, epimerization was observed in both, standard and extract of the spiked samples, after preserving them more than one month as indicated in the experiment section. The formation of other degradation analogues was observed for CTC, which also displayed another peak that also forms epi-analogues. This peak was previously reported and tentatively identified as iCTC based on the absence of the transition $679 \rightarrow$ 444, which indicates the lack of hydroxyl group of the CTC [7]. 
Calibration curves were obtained by spiking soil extracts without TCs and for the entire soil extraction procedure, by fortifying soil samples from control samples with TCs at six concentration levels. Validation results are shown in Table 3. Linear calibration curves were obtained for all compounds and for both soils, with regression coefficients $\left(\mathrm{R}^{2}\right)$ in the range of 0.99 and linear for the complete range tested. Slope values were similar indicating only small differences attributed to the losses of TCs during the extraction procedure. Values obtained for LOD and LOQ for the TCs were in the range $<1-3$ and $3-10 \mu \mathrm{gg}^{-1}$, respectively, which covers the range expected for environmental soil samples and make the analytical method applicable for analysing most of them for the studied TCs.

Mean recoveries and corresponding confidence intervals for the PLE-extraction of six replicate soil samples are listed in Table 4. Recoveries for TCs were between 82 and $99 \%$ and the relative standard deviations (RSDs) on six replicate samples were between 8 and $15 \%$. For the TCs (OTC and CTC) recoveries of approximately $71-78 \%$ were achieved, which is lower than recoveries obtained for the SPE method, indicating that the compounds are not fully extracted from the soil. This is probably due to the many sorption mechanisms involved in the binding of TC to soil, resulting in very strong sorption (Table 1). This corresponds well with previous studies indicating that TCs are sorbed to the clay fraction of the soil by complexation and hydrogen bonding and bound to acid sites in the organic fraction [17-21].

Field study

Frequency, concentration, and identity of TCs found in the soil samples analyzed are outlined in Table 5. Of the 100 samples analyzed, TCs were detected in 25 . The most commonly detected TC was OTC, followed by CTC, TC and DC. OTC was detected in 21 
samples at levels ranging from 15.7 to $105.4 \mu \mathrm{g} \mathrm{kg}{ }^{-1}$, CTC in 11 samples in the concentration range of $5.8-34.4 \mu \mathrm{g} \mathrm{kg}^{-1}$, TC in 9 samples at concentrations from 18.8 to $64.3 \mu \mathrm{g} \mathrm{kg}^{-1}$ and DC in 4 at levels ranging from $12.1-45.7 \mu \mathrm{g} \mathrm{kg}^{-1}$.

On the co-occurrence of TC residues, 12 samples contained two TCs that were in seven cases OTC and CTC, in four OTC and TC and in one OTC and DC. Three samples contained 3 TCs that were OTC, CTC and TC. Only one sample contains the four TCs studied.

The isomerization of the different TCs to their epimers in soil samples was also checked. Data reported in Table 5 were calculated for the sum of both, the TC and the 4-epimer. However, Fig. 5 illustrates a chromatogram of one sample containing OTC and CTC (sample $1 \mathrm{~b}$ of Table 5). The results shows that OTC epimerizes in low percentage, less than $25 \%$, comparing with CTC that shows its isomeric conversion product, iso-CTC and their 4-respective-epimers. As can be observed in the chromatogram, the conversion of CTC to iso CTC also take place in an important percentage. TC is also epimerized in a mean value between OTC and CTC. The quantification of iso-CTC was not possible because we do not have available the iso-CTC standard. These results indicated the need for a further study on TCs metabolites and the difficulties to perform it, starting by the lack of analytical standards.

These data confirm the recent findings reported in the literature [12-15] on that TCs occur in relatively high concentrations and persist in the environment after repeated fertilizations of farmland with liquid manures or sludges. However, these data also include several novelties, such as: (i) the finding of DC; (ii) the observation of co-occurrence of TCs in soil samples that have received an unknown treatment with sludge -this co-occurrence can be by the coexistence of different TCs in the manure or sludge or by the soil treatment with different sludges, and (iii) the different isomerization patterns of TCs in real soil sample. 


\section{Conclusions}

Simultaneous extraction of TC, CTC, OTC and DC from soil was carried out using hot water PLE, clean-up and concentration by SPE and analysis by LC-ESI-MS/MS. Recoveries, LOD and LOQ for the extraction procedure were satisfactory, demonstrating its applicability for simultaneous determination of TCs from soil. It can be concluded that the proposed PLE-SPE method is an interesting alternative extraction technique for the determination of TC residues in soil because it provides similar results to other techniques, reduces the use of organic solvents and complex buffers and does not need $\mathrm{pH}$ adjustment. A pre-concentration and cleanup step is necessary because the large amount of co-extracted humic and fulvic acids. SAX-StrataX was found to be an efficient clean-up step that selectively remove humic and fulvic acids. The proposed method compares well with the results obtained by the other recently reported procedures and present the advantage of eliminating the use of organic solvent as extractants. Considering the savings in time and solvent consumption, which are both diminished by $90 \%$, PLE is an attractive alternative for extracting TC residues from soil.

The application of the method made the detection of significant amounts of persistent TCs in the soil possible. The study pointed out that TCs, which are frequently used worldwide, are persistent in soil in significant amounts, and that these substances represent an actual environmental problem. Ecotoxicological studies, especially on soil microorganisms, should be performed to estimate the risk for the soil flora and the spread of antibiotic resistance.

\section{Acknowledgements}


The authors thank the Spanish Ministry of Science and Innovation together with the European Regional Development Funds (ERDF) (projects GCL2007-66687-C02-01/BOS, CGL2007-66687C02-02 and CGL2008-01693/BTE) and the Conselleria de Sanitat of the Generalitat Valenciana (EVES2008-011) for financial support. P.V.R thanks the Spanish Ministry of Science and Innovation for the FPU grant.

\section{References}

1. Barcelo D (2007) Trac-Trends Anal Chem 26:454-455.

2. Petrovic M and Barcelo D (2007) Trac-Trends Anal Chem 26:486-493.

3. Andreu V, Blasco C, and Pico Y (2007) Trac-Trends Anal Chem 26:534-556.

4. Pico Y and Andreu V (2007) Anal Bioanal Chem 387:1287-1299.

5. Davis JG, Truman CC, Kim SC, Ascough JC, and Carlson K (2006) J Environ Qual 35:22502260 .

6. Kumar K, Gupta SC, Chander Y, and Singh AK (2005) Adv Agron 87:1-54.

7. O'Connor S and Aga DS (2007) Trac-Trends Anal Chem 26:456-465.

8. Kuster M, de Alda MJ, Hernando MD, Petrovic M, Martin-Alonso J, and Barcelo D (2008) J Hydrol 358:112-123.

9. Gros B, Pizzolato TM, Petrovic M, de Alda MJL, and Barcelo D (2008) J Chromatogr A 1189:374-384.

10. Besse JP, Kausch-Barreto C, and Garric J (2008) Hum Ecol Risk Assess 14:665-695.

11. Shlaes DM (2006) Curr Opin Invest Drugs 7:167-171.

12. Hamscher G, Sczesny S, bu-Qare A, Hoper H, and Nau H (2000) Deut Tierarztl Woch 107:332-334.

13. Martinez-Carballo E, Gonzalez-Barreiro C, Scharf S, and Gans O (2007) Environ Pollut 148:570-579. 
14. Hamscher G, Pawelzick HT, Hoper H, and Nau H (2005) Environ Toxicol Chem 24:861-868.

15. Aga DS, O'Connor S, Ensley S, Payero JO, Snow D, and Tarkalson D (2005) J Agric Food Chem 53:7165-7171.

16. De Liguoro M, Cibin V, Capolongo F, Halling-Sorensen B, and Montesissa C (2003) Chemosphere 52:203-212.

17. Sassman SA and Lee LS (2005) Environ Sci Technol 39:7452-7459.

18. Figueroa RA, Leonard A, and Mackay AA (2004) Environ Sci Technol 38:476-483.

19. Pils JRV and Laird DA (2007) Environ Sci Technol 41:1928-1933.

20. Wang YJ, Jia DA, Sun RJ, Zhu HW, and Zhou DM (2008) Environ Sci Technol 42:32543259 .

21. Lutz F and Alber J (2004) Tierarztl Prax 32:180-190.

22. Hamscher G, Sczesny S, Hoper H, and Nau H (2002) Anal Chem 74:1509-1518.

23. Aga DS, Goldfish R, and Kulshrestha P (2003) Analyst 128:658-662.

24. O'Connor S, Locke J, and Aga DS (2007) J Environ Monit 9:1254-1262.

25. Jacobsen AM, Halling-Sorensen B, Ingerslev F, and Hansen SH (2004) J Chromatogr A 1038:157-170.

26. Blasco C, Torres CM, and Pico Y (2007) Trac-Trends Anal Chem 26:895-913.

27. Carretero V, Blasco C, and Pico Y (2008) J Chromatogr A 1209:162-173.

28. Carabias-Martínez R, Rodríguez-Gonzalo E, Revilla-Ruiz P, and Hernández-Méndez J (2005) J Chromatogr A 1089:1-17.

29. de la Cal A, Eljarrat E, and Barcelo D (2003) J Chromatogr A 1021:165-173.

30. Andreu V, Ferrer E, Rubio JL, Font G, and Pico Y (2007) Sci Total Environ 378:124-129.

31. Hamscher G, Priess B, and Nau H (2006) Arch Lebensm Hyg 57:97-101.

32. Bogialli S, Curini R, Di Corcia A, Lagana A, and Rizzuti G (2006) J Agric Food Chem 54:1564-1570. 
Table 1. LC-MS/MS conditions for confirming and quantifying the selected tetracyclines in soil samples ${ }^{\mathrm{a}}$

\begin{tabular}{|c|c|c|c|c|c|c|c|c|}
\hline Compound & $\mathrm{t}_{\mathrm{r}}(\min )$ & $\begin{array}{l}\text { Quantifier SRM } \\
(\mathrm{m} / \mathrm{z})\end{array}$ & $\begin{array}{c}\mathrm{CV}^{\mathrm{b}} \\
(\mathrm{V})\end{array}$ & $\begin{array}{l}\mathrm{CE}^{\mathrm{c}} \\
(\mathrm{V})\end{array}$ & Qualifier SMR (m/z) & $\begin{array}{c}\mathrm{CV}^{\mathrm{b}} \\
(\mathrm{V})\end{array}$ & $\begin{array}{c}\mathrm{CE}^{\mathrm{c}} \\
(\mathrm{V})\end{array}$ & Ion ratios $^{\mathrm{d}}$ \\
\hline $\mathrm{TC}$ & 8.23 & $445 \rightarrow 410$ & 18 & 12 & $445 \rightarrow 427$ & 18 & 18 & $0.536 \pm 0.003$ \\
\hline e-TC & 6.23 & $445 \rightarrow 410$ & 18 & 12 & $445 \rightarrow 427$ & 18 & 18 & $0.502 \pm 0.008$ \\
\hline CTC & 10.72 & $479 \rightarrow 444$ & 20 & 15 & $479 \rightarrow 444$ & 20 & 18 & $1.425 \pm 0.009$ \\
\hline e-CTC & 9.11 & $479 \rightarrow 444$ & 20 & 15 & $479 \rightarrow 444$ & 20 & 18 & $0.729 \pm 0.007$ \\
\hline OTC & 8.66 & $461 \rightarrow 443$ & 18 & 10 & $461 \rightarrow 426$ & 18 & 18 & $0.358 \pm 0.003$ \\
\hline e-OTC & 7.53 & $461 \rightarrow 443$ & 18 & 10 & $461 \rightarrow 426$ & 18 & 18 & $0.504 \pm 0.004$ \\
\hline DMC (IS) & 9.38 & $465 \rightarrow 448$ & 22 & 15 & $465 \rightarrow 430$ & 22 & 18 & $0.407 \pm 0.010$ \\
\hline $\mathrm{DC}$ & 12.60 & $445 \rightarrow 428$ & 20 & 18 & $445 \rightarrow 321$ & 20 & 30 & $0.060 \pm 0.001$ \\
\hline
\end{tabular}

${ }^{a}$ The criteria for residue identification were (i) four identification points through the measurement of two product ions plus the precursor ion; (ii) retention time of suspected analyte and reference standard within the tolerance interval of $\pm 2.5 \%$, and (iii) the ion ratio for each analyte in samples matches that of the standards within the maximum permitted tolerances $( \pm 20 \%$ for TC, e-TC, CTC. e-CTC and e-OTC; \pm 25 for OTC, and \pm 50 for DC).

${ }^{\mathrm{b}} \mathrm{CV}=$ cone voltage

${ }^{\mathrm{c}} \mathrm{CE}=$ collision energy

${ }^{\mathrm{d}}$ Ion ratios $=$ the ratio of the intensities of the two most abundant transitions of each tetracycline determined from the analysis of standards prepared in methanol-water $(10: 90 \mathrm{v} / \mathrm{v})(n=15)$. 
Table 2. Linear regression parameters of TCs from soil calibration curves ${ }^{\mathrm{a}}$ ranging from 10 to $500 \mu \mathrm{g} \mathrm{kg}^{-1}$ (6 points, triplicate analyses), LODs and LOQs

\begin{tabular}{|c|c|c|c|c|c|c|c|c|}
\hline \multirow[t]{2}{*}{ Compound } & \multicolumn{3}{|c|}{ Spiked soil extracts } & \multicolumn{3}{|c|}{ Entiere soil extraction procedure } & \multirow{2}{*}{$\begin{array}{l}\text { LODs } \\
\mu \mathrm{g} \mathrm{kg}^{-1}\end{array}$} & \multirow{2}{*}{$\begin{array}{l}\text { LOQs } \\
\mu \mathrm{g} \mathrm{kg}^{-1}\end{array}$} \\
\hline & Slope & $y$-intercept & $R^{2}$ & Slope & $y$-intercept & $R^{2}$ & & \\
\hline $\mathrm{TC}$ & 29.85 & 12.75 & 0.9985 & 27.25 & 28.25 & 0.9989 & 1 & 3 \\
\hline OTC & 20.12 & -4.74 & 0.9983 & 18.62 & 18.01 & 0.9984 & 2 & 6 \\
\hline СТC & 10.45 & 14.28 & 0.9998 & 8.94 & 14.95 & 0.9996 & 3 & 10 \\
\hline DC & 24.20 & 13.94 & 0.9996 & 21.09 & 19.54 & 0.9998 & 1 & 3 \\
\hline e-TC & 28.30 & -19.07 & 0.9996 & 25.82 & 12.01 & 0.9990 & 1 & 3 \\
\hline e-OTC & 26.42 & -12.73 & 0.9984 & 24.42 & 9.95 & 0.9972 & 2 & 6 \\
\hline e-CTC & 15.62 & 25.73 & 0.9971 & 13.09 & -24.83 & 0.9988 & 3 & 10 \\
\hline
\end{tabular}

${ }^{a}$ The linear regression analysis was carried out by plotting the peak area ratio of analyte and IS versus the analyte concentration 
Table 3. Recoveries and the relative standard deviations (RSDs) of the studied TCs in soil spiked at different concentrations.

\begin{tabular}{lcccccc}
\hline TCs & \multicolumn{6}{c}{ Concentration } \\
\cline { 2 - 7 } & \multicolumn{2}{c}{$10 \mu \mathrm{g} \mathrm{kg}^{-1}$} & \multicolumn{2}{c}{$50 \mu \mathrm{g} \mathrm{kg}^{-1}$} & \multicolumn{2}{c}{$500 \mu \mathrm{g} \mathrm{kg}^{-1}$} \\
\cline { 2 - 7 } & recovery (\%) & RSD (\%) & recovery (\%) & RSD (\%) & recovery (\%) & RSD (\%) \\
\hline TC & 86.7 & 14.8 & 93.8 & 8.6 & 96.0 & 10.8 \\
OTC & 74.2 & 12.3 & 73.7 & 10.6 & 77.3 & 10.2 \\
CTC & 75.9 & 13.1 & 76.5 & 11.1 & 76.4 & 9.0 \\
DC & 84.2 & 14.5 & 83.2 & 8.5 & 92.1 & 8.5 \\
e-TC & 83.2 & 14.5 & 83.2 & 8.0 & 92.1 & 8.5 \\
e-OTC & 73.0 & 11.6 & 71.2 & 8.6 & 75.7 & 7.7 \\
e-CTC & 71.2 & 12.5 & 73.2 & 8.5 & 72.1 & 8.5 \\
\hline
\end{tabular}

${ }^{a}$ Values are the mean of five independent determinations at each concentration. 
Table 4. Results of the investigation of 100 samples collected of the Ap horizon $(0-20 \mathrm{~cm})$ from 50 fields located in the South surrounding of Valencia city in February 2007.

\begin{tabular}{|c|c|c|c|c|}
\hline \multirow[t]{2}{*}{ Sample $^{\mathrm{a}}$} & \multicolumn{4}{|c|}{$\mathrm{TCs}^{\mathrm{b}}($ mean $\pm \mathrm{SD}, n=4)$} \\
\hline & OTC, $\mu \mathrm{g} \mathrm{kg}^{-1}$ & $\mathrm{CTC}, \mu \mathrm{g} \mathrm{kg}^{-1}$ & $\mathrm{TC}, \mu \mathrm{g} \mathrm{kg}^{-1}$ & $\mathrm{DC}, \mu \mathrm{g} \mathrm{kg}^{-1}$ \\
\hline $1 \mathrm{a}$ & $25.0 \pm 5.3$ & $10.4 \pm 0.2$ & & \\
\hline $1 b$ & $32.1 \pm 3.2$ & $8.1 \pm 2.1$ & & \\
\hline $4 a$ & $22.4 \pm 2.1$ & $12.9 \pm 4.8$ & $62.7 \pm 6.1$ & \\
\hline $4 \mathrm{~b}$ & $34.2 \pm 6.4$ & $15.2 \pm 4.4$ & $64.3 \pm 7.2$ & \\
\hline $7 \mathrm{a}$ & $29.6 \pm 4.3$ & & & \\
\hline $10 \mathrm{a}$ & $52.2 \pm 10.1$ & $7.1 \pm 0.3$ & & \\
\hline $10 \mathrm{~b}$ & $45.7 \pm 9.3$ & $12.8 \pm 0.6$ & & \\
\hline $15 b$ & $32.2 \pm 4.4$ & $10.3 \pm 1.2$ & $54.5 \pm 7.8$ & $12.1 \pm 1.2$ \\
\hline $17 \mathrm{a}$ & & & & $45.2 \pm 4.3$ \\
\hline $17 \mathrm{~b}$ & & & & $43.4 \pm 5.8$ \\
\hline $20 \mathrm{a}$ & $15.7 \pm 1.3$ & & & \\
\hline $20 \mathrm{~b}$ & $32.3 \pm 5.0$ & & & \\
\hline $23 \mathrm{a}$ & & & & \\
\hline $30 \mathrm{a}$ & $105.4 \pm 12.8$ & $14.3 \pm 0.4$ & & \\
\hline $30 \mathrm{~b}$ & $95.8 \pm 11.6$ & $5.8 \pm 0.2$ & & \\
\hline $42 b$ & & & $34.0 \pm 2.7$ & \\
\hline $49 b$ & $34.5 \pm 3.3$ & & & $45.7 \pm 8.2$ \\
\hline $57 \mathrm{a}$ & $45.2 \pm 6.2$ & & $29.4 \pm 5.2$ & \\
\hline $57 \mathrm{~b}$ & $52.2 \pm 7.7$ & & $18.8 \pm 4.1$ & \\
\hline $72 \mathrm{a}$ & $22.7 \pm 8.1$ & & & \\
\hline $72 b$ & $25.4 \pm 6.4$ & & & \\
\hline $83 a$ & $67.6 \pm 5.2$ & $10.3 \pm 0.6$ & $42.1 \pm 3.8$ & \\
\hline $94 a$ & $34.5 \pm 3.2$ & & $22.3 \pm 4.3$ & \\
\hline $94 b$ & $28.5 \pm 2.3$ & & $32.6 \pm 3.8$ & \\
\hline $97 b$ & $92.9 \pm 12.0$ & $34.4 \pm 0.9$ & & \\
\hline
\end{tabular}

${ }^{a}$ The two samples taken of each field were identified by the same number and the letter "a" or "b".

${ }^{b}$ The concentration of each TCs is the sum of the TC and the 4-epimer if exists 


\section{LEGEND OF FIGURES}

Fig. 1 Efficiency of the PLE system using different extractants. Soil samples were spiked at $50 \mu \mathrm{g} \mathrm{kg}^{-1}$ and the extract were analyzed by LC-ESI-MS/MS without further cleanup step.

Fig 2. Efficiency on the SPE clean-up using different SPE platforms at two different concentrations 1.25 and $12.5 \mu \mathrm{g} \mathrm{l}^{-1}$.

Fig. 3 LC-MS/MS chromatograms of soil spiked at $10 \mu \mathrm{g} \mathrm{kg}^{-1}$ of each tetracycline including the internal standard (A) TIC of the ten precursor $\rightarrow$ product ion transitions reported in Table 1, (B) mass chromatogram for DC (445 $\rightarrow 428$ transition), (C) mass chromatogram for TC (445 $\rightarrow 427$ transition), (D) mass chromatogram for OTC $(461 \rightarrow$ 443 transition) and (E) mass chromatogram for CTC (479 $\rightarrow 444$ transition).

Fig. 4 LC-MS/MS chromatograms of soil spiked at $10 \mu \mathrm{g} \mathrm{kg}^{-1}$ of each tetracycline epimer and the the internal standard (A) TIC of the ten precursor $\rightarrow$ product ion transitions reported in Table 1, (B) mass chromatogram for e-TC (445 $\rightarrow 427$ transition), (C) mass chromatogram for e-OTC (461 $\rightarrow 443$ transition) and (D) mass chromatogram for e-CTC $(479 \rightarrow 444$ transition $)$

Fig. 5 LC-MS/MS chromatogram corresponding to sample $1 b$. 
Figure 1

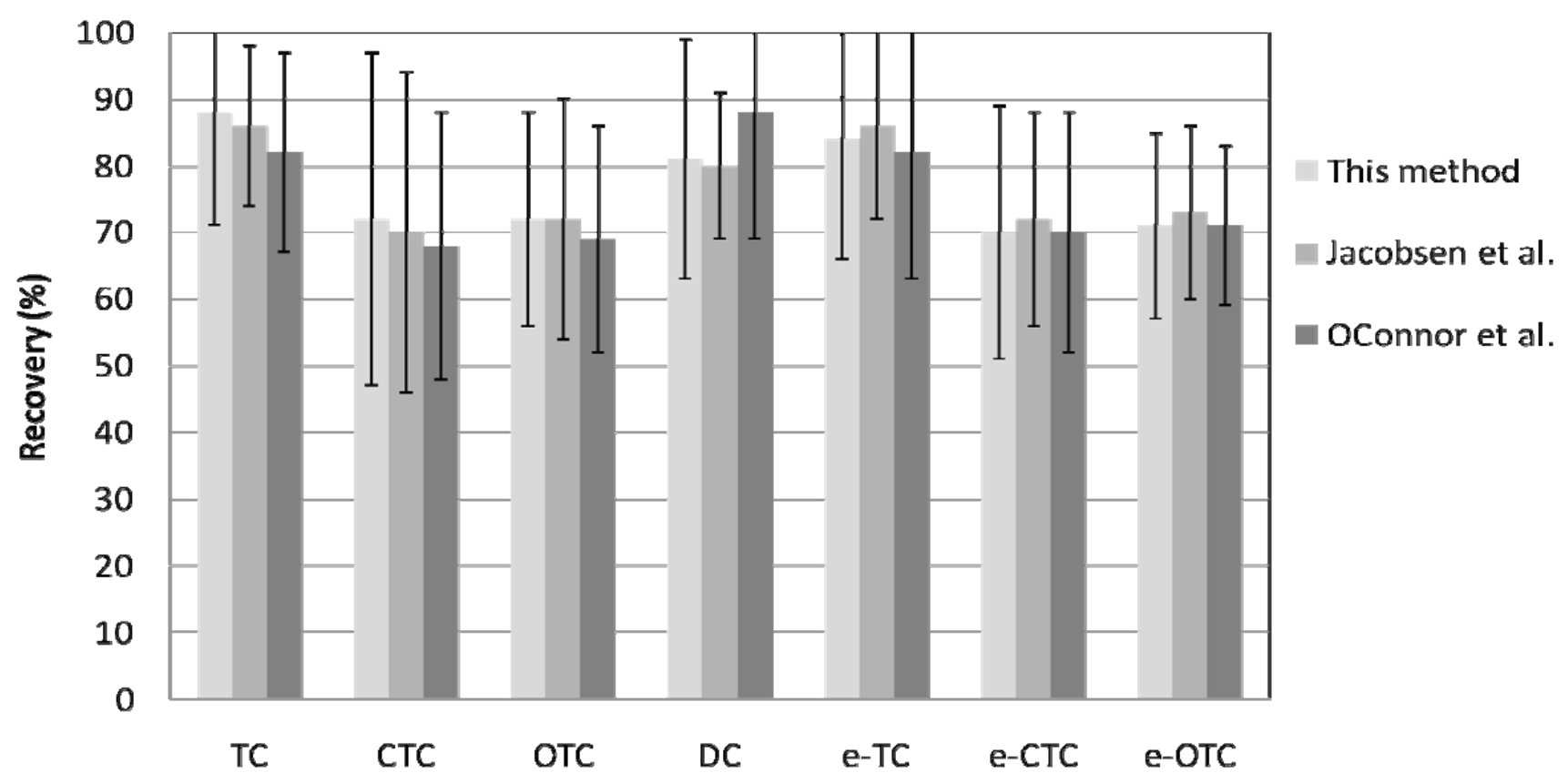


Figure 2

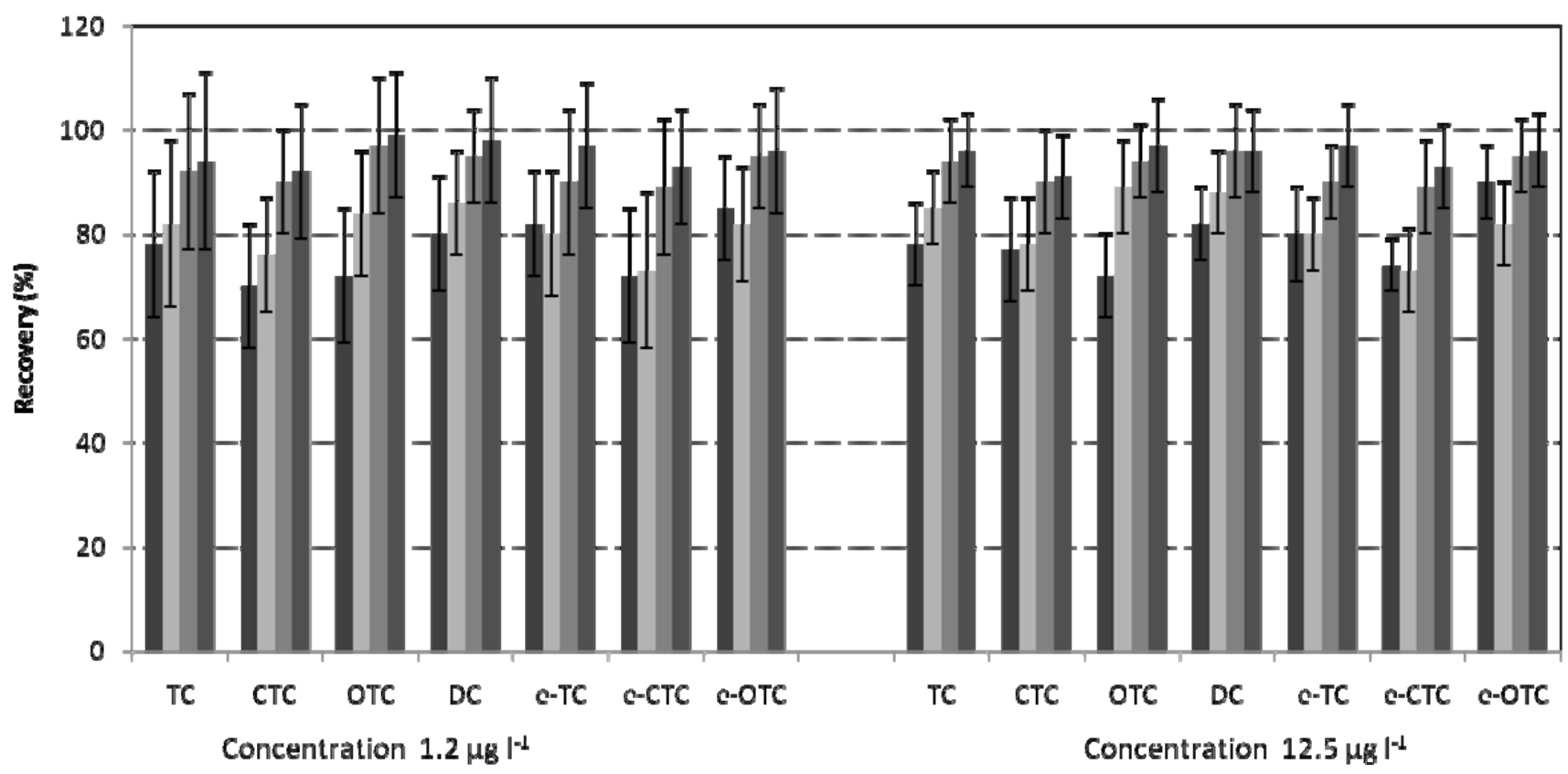

- HLB

StrataX

- SAX-IILB

- SAX-Strate 
Figure 3

Soil sample spiked with $10 \mu \mathrm{g} \mathrm{kg}^{-1}$ of each TC and of the IS

RecoverySoil 5

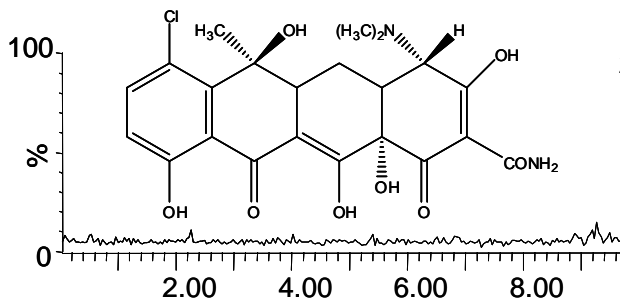

10.72

$272]$ CTC
MRM of 10 Channels ES+

$479>444$

$1.06 \mathrm{e} 3$

E Area

RecoverSoil 5

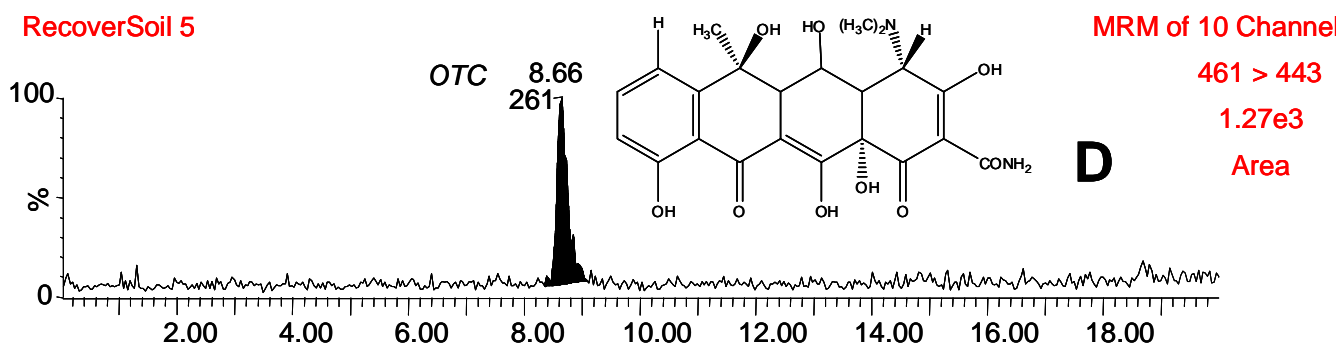

RecoverySoil 5

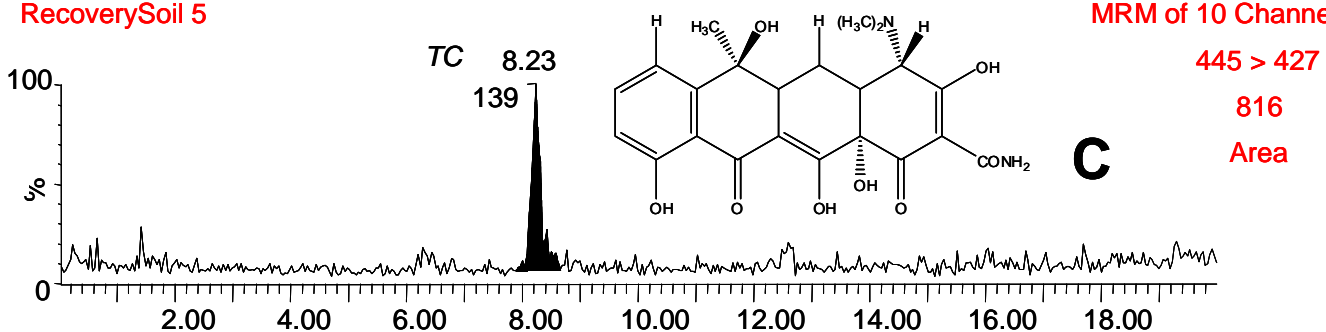

$\begin{array}{lllllllll}2.00 & 4.00 & 6.00 & 8.00 & 10.00 & 12.00 & 14.00 & 16.00 & 18.00\end{array}$

RecoverySoil 5

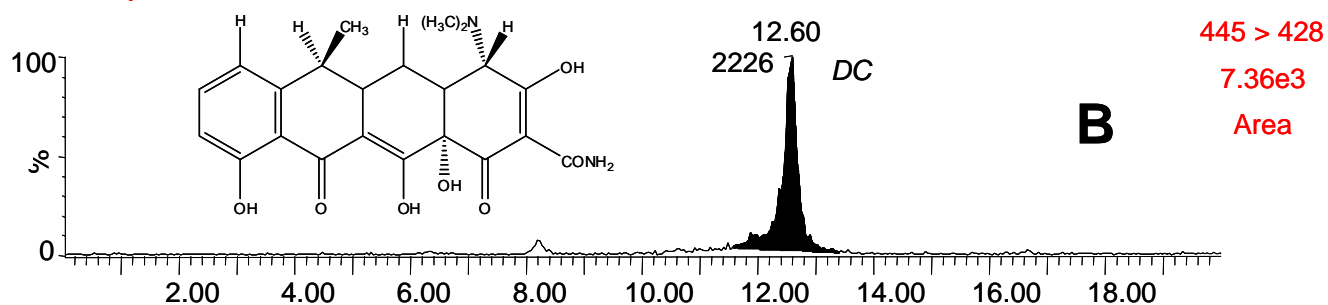

RecoverySoil 5

$$
D M C \text { (IS) }
$$

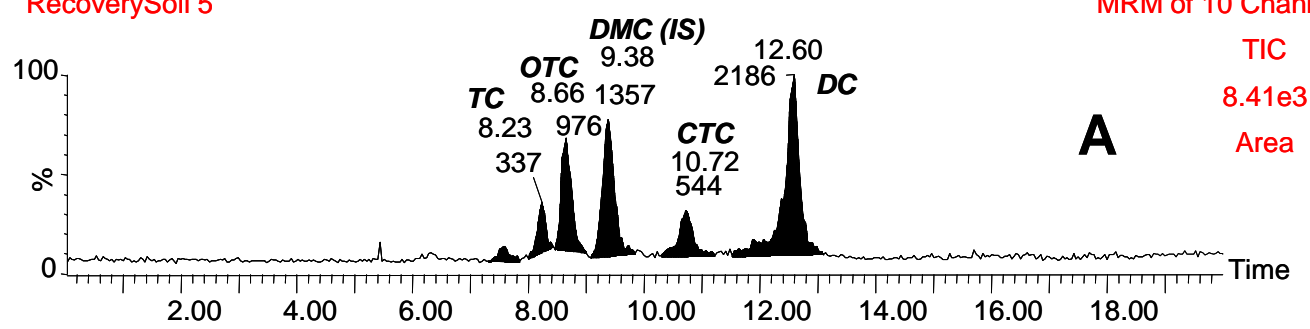


Figure 4

Soil sample spiked with10 $\mu \mathrm{gg}^{-1}$ of each e-TC and of the IS

epi-RecoverySoil 5

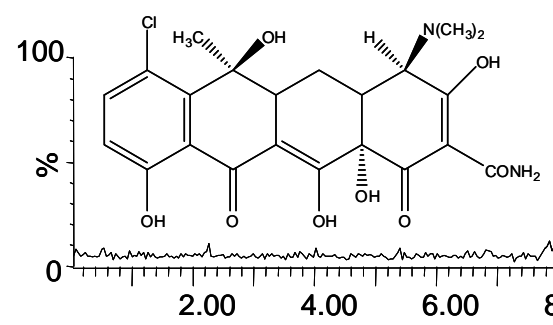

epi-RecoverySoil 5

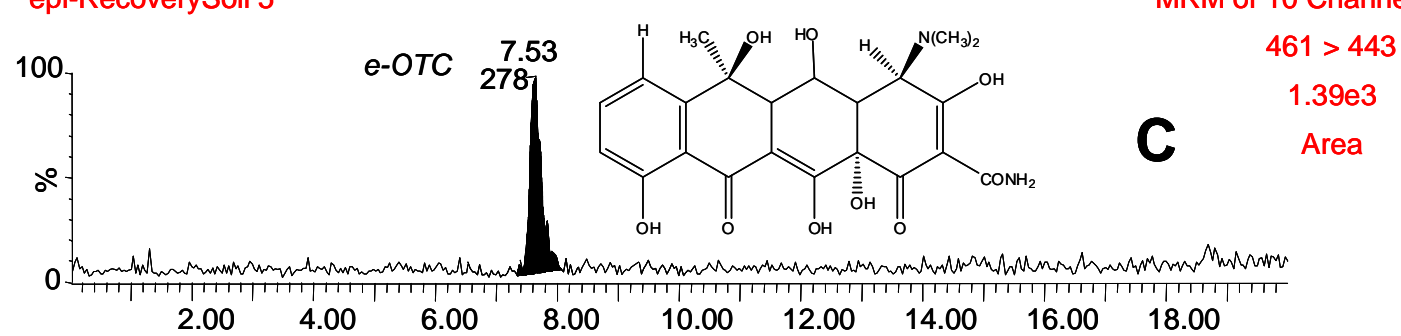

MRM of 10 Channels ES+ $479>444$

$1.15 \mathrm{e} 3$

D Area
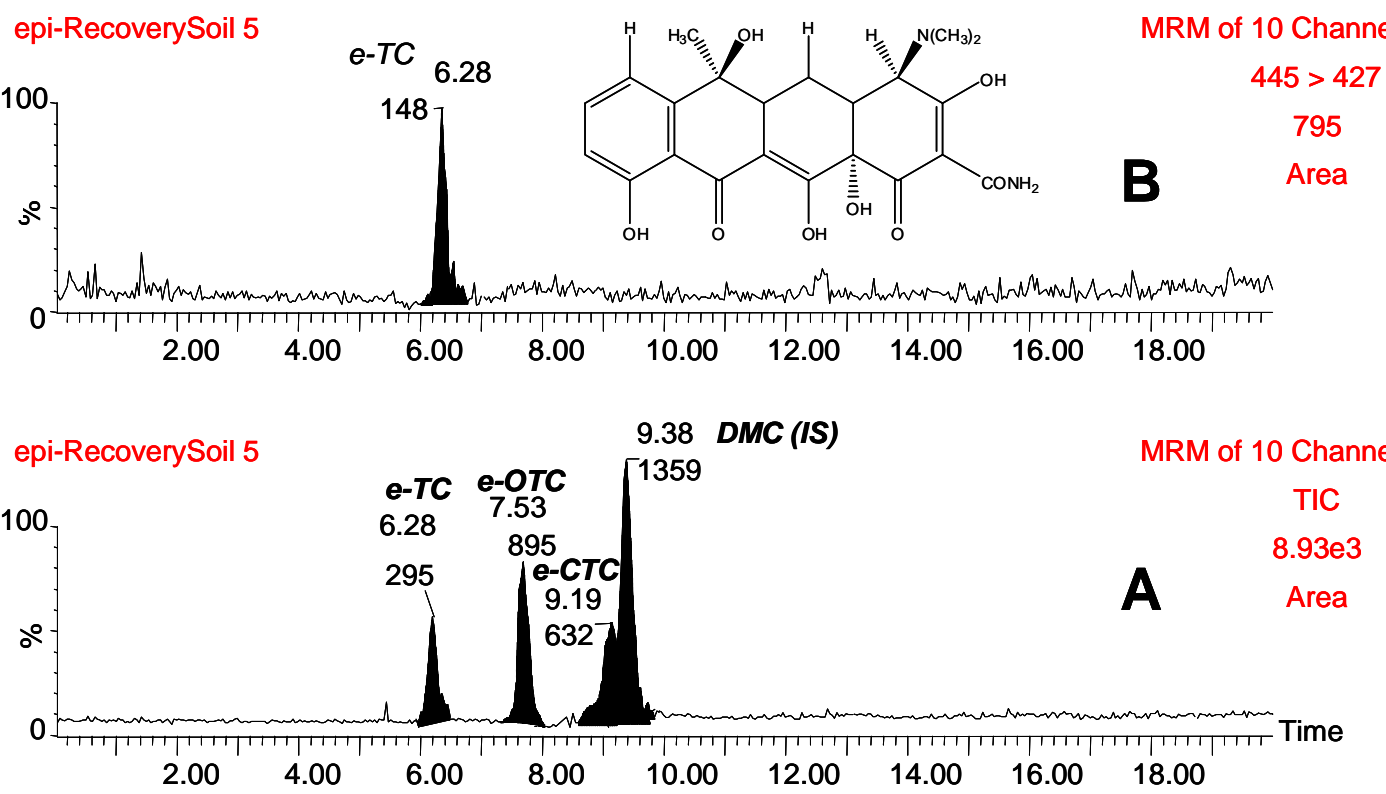
Figure 5

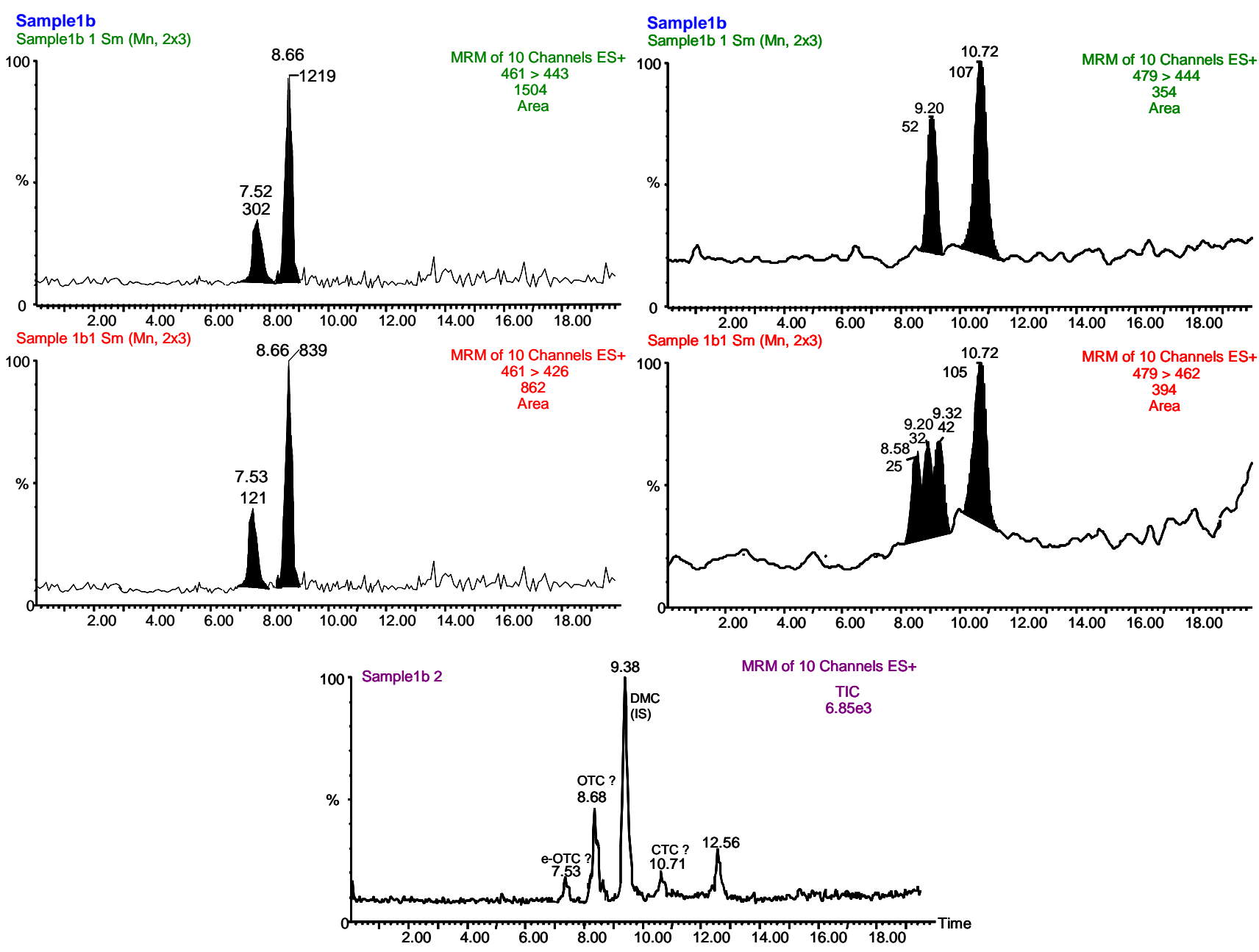

